\title{
Fire incidents visualization and pattern recognition using machine learning algorithms
}

\author{
Jonardo R. Asor ${ }^{1}$, Jefferson L. Lerios ${ }^{2}$, Sherwin B. Sapin ${ }^{3}$, Jocelyn O. Padallan ${ }^{4}$, \\ Chester Alexis C. Buama ${ }^{5}$ \\ ${ }^{1-4}$ College of Computer Studies (CCS), Laguna State Polytechnic University, Los Baños, Laguna, Philippines \\ ${ }^{5}$ College of Arts and Sciences (CAS), Laguna State Polytechnic University, Los Baños, Laguna, Philippines
}

\begin{tabular}{l} 
Article Info \\
\hline Article history: \\
Received Jan 13, 2021 \\
Revised May 31, 2021 \\
Accepted Jun 13, 2021 \\
\hline
\end{tabular}

\section{Keywords:}

Fire

Model development

Pattern recognition

RNN

\begin{abstract}
A fire incident is a devastating event that can be avoided with enough knowledge on how and when it may occur. For the past years, fire incidents have become a big problem for the Philippines, since it affects the socioeconomic growth of the country. Machine learning algorithm is a well-known technique to predict and analyze data. It can also be used to recognize pattern and develop models for artificial intelligence. Pattern recognition through machine learning algorithm is already established and have proven itself accurate in different fields such as education, crime, health and many others including fire incidents. This paper aims to develop a model for recognizing patterns of fire incidents in the province of Laguna, Philippines implementing a machine learning algorithm. With the foregoing project, it is found out that a recurrent neural network shows an astonishing result in terms of pattern recognition. Further, it is also found that Calamba City is the most vulnerable area in case of fire occurrence in the Province of Laguna.
\end{abstract}

This is an open access article under the CC BY-SA license.

\section{Corresponding Author:}

Jonardo R. Asor

College of Computer Studies

Laguna State Polytechnic University

Malinta, Los Baños, Laguna 4030, Philippines

Email: asor.jonardo@lspu.edu.ph

\section{INTRODUCTION}

Fire is one of the initial discoveries of humanity, and arguably the most important one. It gave us the ability to cook food, forge metal tools, and manage power plants. The known hazards of fire for the first generations for the people were limited to thermal risks only. But despite the essential use of light, it can destroy houses and properties in less than an hour [1]. Fire disaster is a common threat to lives and property. The effects of fire disasters can be harmful to humans; it can lead to severe injury and even death. The majority of fatalities are trapped civilians who died of smoke inhalation of burning materials [2].

Fire-related injuries and deaths are growing increasingly and need to be controlled. Research in London, England has shown that the majority of fire deaths have occurred in communities with higher levels of socioeconomic deprivation, measured by unemployment, low incomes, health education, crime and housing [3]. The 2018 report of World Health Organization (WHO) regarding loss of life related to fire incidents shows that the Philippines ranks 11th out of 20 in the ranking of most death tolls caused by fire incidents with approximately 1000 fatalities recorded [4]. Physical discomfort and overexertion, crashes, objects being hit, and exposure to fire materials are the main causes of fire injury [5].

In the Philippines especially in the National Capital Area, fire incidents have increased significantly over the years and are major threat to the economy. Fires spread rapidly due to adjoining homes in residential 
areas. Many factors that lead to fire-related accidents are triggered by electrical wiring, unattended gas stoves and the use of candles as a light source. Meanwhile, unauthorized connections and the use of cable jumpers for electric meters are the number one cause of fire in crowded residential areas [6]. The need for technical improvements in the study of fire events is very important. With the aid of additional fire analysis equipment, fire services can have a greater understanding of fire incidents. They would then be able to come to a conclusion about how to minimize and prevent fire accidents. Fire Departments will carry out a risk-reduction, drill, programs, and awareness-raising plan [7].

Pattern recognition is a scientific discipline whose aim is to recognize objects in a variety of categories or classes. Depending on the application, these objects may be categorized as images or signal waveforms or some sort of measurement that needs to be classified [8] and referred to these objects using the generic term patterns [9]. Pattern identification has a long tradition, but until the 1960s, it was mainly the productivity of academic study in the field of statistics [10], [11]. As with all else the advent of computers increased the demand for practical applications for pattern recognition, which in turn generated new demands for more theoretical developments[12].

Regression comes from the category of supervised learning; deals with the relationship between a categorical dependent variable and one or more independent variables by estimating probabilities using a sigmoid function [13]. In other instances, analysis uses the decision tree algorithm. Fire accidents in terms of decision-making principles, using the terminology of decision-making and risk analysis as the basis for the relationship between fire management decision-making and incident outcomes [14]. The basic approach is embodied in one of the core principles of decision analysis, that of the decomposition by which large, complex problems can be better understood by breaking them down or "decomposing" them into smaller more manageable problems that can be resolved or defined in some detail [15]. Today, machine learning algorithms are commonly used to identify trends not only in crime, education, health and business but also in fire incidents [16]-[18]. The main objective of this paper is to recognized pattern and identify trends of fire incidents in Laguna, Philippines using machine learning algorithms.

\section{RESEARCH METHOD}

\subsection{Data collection}

The fire incidents data obtained from Bureau of fire protection (BFP) are records covering the period of 2014 to 2018 fire incidents in Laguna. The data are classified with the following categories; time and place where the fire incident occurred, occupancy, the cause of fire, alarm level, and casualties in the fire incident. Alarm level will be used as the class for classifying fire incidents. It is composed of six instances namely: $1^{\text {st }}$, $2^{\text {nd }}, 3^{\text {rd }}, 4^{\text {th }}, 5^{\text {th }}$ and no alarm.

\subsection{Pre-processing of data}

Pre-processing is necessary to make sure that the dataset contains no biases in recognizing patterns of fire incidents. First, the data underwent normalization where the data has an unknown or unidentified attribute and is not compatible with the specific dataset. The normalization process is required to minimize data redundancy. Cleaning and organizing the data (removing symbols and converting the text into lowercase) is also recommended to make the data meaningful. The fire incidents data has numerous attributes in the given raw data which are not needed in developing the project. In assuring that a quality model will be developed, attributes with the same meaning was removed [19], [20]. Since date occurred and incident report are the same, one of these attributes was removed and the other one was sustained to remove data noises. This was done in the entire dataset which provide the dataset attributes shown in Table 1.

Table 1. Attributes of Laguna fire incidents dataset

\begin{tabular}{lll}
\hline \multicolumn{1}{c}{ Attributes } & \multicolumn{1}{c}{ Description } & Data type \\
\hline Date Occurred & Exact Date of Fire Incident happen & Date \\
Time Occurred & Exact Time & Time \\
Occupancy & Classification of Structure & String \\
Cause of Fire & Causes of the Fire & String \\
Alarm Level & The Alarm Level of Fire & Integer \\
Casualties & The casualties made by the Fire & Integer \\
Location & Where the fire incidents occurred & String \\
\hline
\end{tabular}

In statistics and machine learning, it is usually a requirement to split the data into two subsets-the train and test set. The train dataset will be used to fit the model, in order to make predictions on the test dataset. 
Separating the data into train set which is $80 \%$ and $20 \%$, the test set. These two are needed so there is an unbiased prediction of fire incidents data and evaluate data mining models [21]. By simply looking at Table 2, it is noticeable that the datasets contain so much bias in terms of numbers of occurrences. Since this project intends to recognize pattern, this will not be a problem, however, in some studies especially intelligent systems and model development, this bias must be eliminated. Nonetheless, synthetic minority oversampling technique (SMOTE) is implemented in this paper to assure that the evaluation of machine learning algorithm will not contain any bias. SMOTE finds the closest neighbors in minority class for each sample in the class. It then draws a line between the neighbors and generates random points on the line for producing new data. The use of this method is vital in predicting class of fire incidents [22].

Table 2. Distribution of fire alarm in train and test dataset

\begin{tabular}{ccc}
\hline Alarm Level & Train Set & Test Set \\
\hline $1^{\text {st }}$ Alarm & 2,098 & 524 \\
$2^{\text {nd }}$ Alarm & 53 & 13 \\
$3^{\text {rd }}$ Alarm & 19 & 5 \\
$4^{\text {th }}$ Alarm & 9 & 2 \\
$5^{\text {th }}$ Alarm & 1 & 1 \\
No Alarm & 106 & 27 \\
Total & 2286 & 57 \\
\hline
\end{tabular}

\subsection{Algorithm evaluation}

In order to understand the comparative qualities of the algorithm, it is crucial to evaluate them. The primary methods to evaluation can be characterized as experimental. Experimental evaluation applies the algorithm to learning tasks to study its performance in practice. Some models may give satisfying results when evaluating but others may give poor results when evaluated [23]. Therefore, it is an excellent way to evaluate machine learning algorithms to have a well processed project. This phase requires the following:

\subsubsection{Confusion matrix}

A confusion matrix will be used to characterize the output of a classification model or classifier in a collection of test data for which true values are known. This makes quick recognition of confusion between groups. One class is usually mismarked as the other, so most output metrics are computed from the confusion matrix [24], [25]. The confusion matrix is useful for evaluating a model when knowing the true response values. The point of the confusion matrix in the project is to define the performance such as measuring precision, recall, f-score, and fallout, as shown in Figure 1 [26], [27].

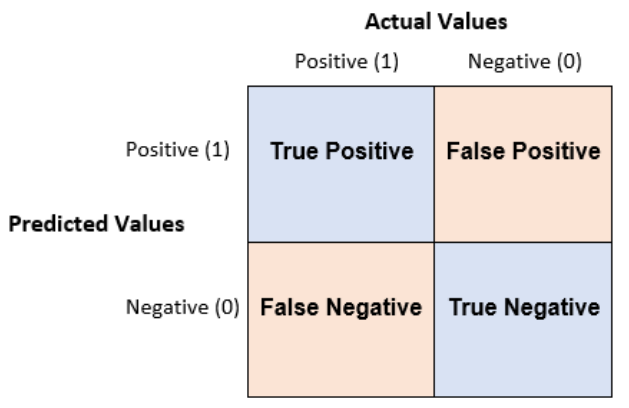

Figure 1. Confusion Matrix

\subsubsection{Recall/sensitivity}

The recall metric shows the capability of a model to discover all the appropriate cases in a dataset. It is the ratio of the total number of suitable classified positive examples divided by the total number of positive examples. It can be thought as a model's skill to find all the data points of interest in a dataset. The (1) show the metric formula of recall.

$$
\text { Recall }=\frac{T P}{T P+F N} \frac{T P}{T P+F N}
$$

\subsubsection{Precision}

The precision metric shows the accuracy of the positive class. It also measures how possible the prediction

Fire incidents visualization and pattern recognition using machine learning ... (Jonardo R. Asor) 
of the positive class is accurate. Precision is identified as the number of true positives divided by the number of true positive plus the number of false positives. False positives are cases that the model incorrectly labels as positive that are actually negative. The (2) shows the metric formula of precision.

$$
\text { Precision }=\frac{T P}{T P+F P} \frac{T P}{T P+F P}
$$

\subsubsection{F-Score}

In some conditions, it is remarkable to maximize the use of either recall or precision at the cost of the other metrics. F-score is the harmonic mean of both precision and recall, whereas for an F-score scope the best value is 1 and the worst is 0 . It gives the weighted average of recall and precision. There are other metrics for combining precision and recall, but the $\mathrm{F}$-score is the most commonly used. If creating a balanced classification model with the optimal balance of recall and precision, then the use of F-score is an excellent choice. The (3) shows the metric formula of F-score.

$$
\text { F-Score }=2 * \frac{\text { Precision } * \text { Recall }}{\text { Precision }+ \text { Recall }}
$$

\subsubsection{Specificity}

It quantifies the evasion of false positives. It is called true negative rate, measuring the proportion of negatives that are correctly identified. In this study, specificity will measure how many correct alarm levels are predicted correctly. The (4) shows the metric formula of specificity.

$$
\text { Specificity }=\frac{T N}{T N+F P}
$$

\subsubsection{Fallout}

The false positive rate or fallout is calculated as the ratio between the number of negative events wrongly categorized as positive and the total number of actual negative events. Fallout is required to compute and illustrate the two metrics to have a better representation of the receiver operating characteristic (ROC) curve. The (5) shows the metric formula of fallout.

$$
\text { Fallout }=1-\text { Specificity }
$$

\section{RESULTS AND DISCUSSION}

In this study four different machine learning algorithms namely, support vector regressor, decision tree regressor, random forest regressor and recurrent neural network (RNN) were used to developed a model for pattern recognition and visualization of fire occurrences in the province of Laguna, Philippines. This section will discuss the findings in both model development and data visualization. Figures and tables were used to easily show the results in experiments done in this study.

\subsection{Results based on algorithm evaluation}

As shown in Table 3, all of the algorithms passed the typical accuracy for model development, however, as shown on the table, Decision tree regressor and random forest regressor overfits the model which was not a great idea. Furthermore, RNN shows an impressive result but has a minor problem in terms of the distance result of root mean square error (RMSE) and standard deviation. This leads to do further evaluation in the learning of each algorithm.

Table 3. Root mean squared error report of algorithms

\begin{tabular}{lcccc}
\hline \multicolumn{1}{c}{ Algorithm } & RMSE (train) & RMSE (test) & STD (train) & STD (test) \\
\hline Support Vector Regressor & 0.62 & 0.62 & 0.23 & 0.24 \\
Decision Tree Regressor & 1.69 & 1.66 & 0.25 & 0.23 \\
Random Forest Regressor & 0.13 & 7.73 & 0.25 & 0.31 \\
Recurrent Neural Network & 0.092 & 0.085 & 0.162 & 0.171 \\
\hline
\end{tabular}

Table 4 represents the confusion matrix which is acquired through the use of Python and after applying SMOTE. It includes true positive, true negative, false positive and false negative of the label chosen in the dataset. Hence, the table shows that recurrent neural network only predicted a wrong label in $2^{\text {nd }}$ alarm and no alarm. Therefore, there are some values which may be found on $1^{\text {st }}$ alarm and no alarm. 
Table 4. Confusion matrix of RNN based on the fire alarm levels

\begin{tabular}{|c|c|c|c|c|c|}
\hline & True $1^{\text {st }}$ Alarm & True $2^{\text {nd }}$ Alarm & True $3^{\text {rd }}$ Alarm & True $4^{\text {th }}$ Alarm & True No Alarm \\
\hline Predicted $1^{\text {st }}$ Alarm & 116 & 0 & 0 & 0 & 0 \\
\hline Predicted $2^{\text {nd }}$ Alarm & 0 & 120 & 0 & 0 & 8 \\
\hline Predicted $3^{\text {rd }}$ Alarm & 0 & 0 & 129 & 0 & 0 \\
\hline Predicted $4^{\text {th }}$ Alarm & 0 & 0 & 0 & 120 & 0 \\
\hline Predicted No Alarm & 4 & 0 & 0 & 0 & 112 \\
\hline
\end{tabular}

As shown in Table 5, recall has a total mean of 0.98 which means that the classified labels are correct with minimum error. Likewise, as indicated in precision, the correctness of the algorithm in terms of predicting fire occurrence is accurate gaining a total mean score of 0.982 . F-score garnered a total mean of 0.98 approaching 1 which is the base of prediction accuracy. As for specificity, 0.982 total mean was acquired which means that the algorithm is capable of specifying each label in classification correctly. Whereas, fallout which represents the incorrect prediction of the algorithm, a total mean of 0.02 was attained, therefore, the algorithm has a promising prediction capability.

Table 5. Result of RNN algorithm evaluation

\begin{tabular}{lccccc}
\hline Alarm Levels & Recall & Precision & F-Score & Specificity & Fallout \\
\hline $1^{\text {st }}$ Alarm & 0.97 & 1 & 0.98 & 0.97 & 0.03 \\
$2^{\text {nd }}$ Alarm & 1 & 0.94 & 0.97 & 1 & 0 \\
$3^{\text {rd }}$ Alarm & 1 & 1 & 1 & 1 & 0 \\
$4^{\text {th }}$ Alarm & 1 & 1 & 1 & 1 & 0 \\
No Alarm & 0.93 & 0.97 & 0.95 & 0.94 & 0.06 \\
Total Mean & 0.98 & 0.982 & 0.98 & 0.982 & 0.02 \\
\hline
\end{tabular}

\subsection{Results based on data visualization}

Based on the data of BFP, most cases of fire occurrences in Laguna were during the year 2014 with 802 cases while 2017 has the least occurrences accumulated with 449 cases as shown in Figure 2 . The changes over time in the graph is dynamic, there are years that sometimes the cases of incidents are enormous and sometimes, lesser. Figure 3 shows that the highest case of fire incidents occurred in the month of April having 627 incidents and taking the closest spot in terms of incidents recorded is the month of March, recording 618 events. Moreover, the month of September has the least occurrence of fire incidents accumulating 77 cases in a 5 years span from 2014 to 2018 .

Figure 4 displays that fire incidents usually happen during the weekends. The graph signifies the numbers of fire events that occurs every day in the province Laguna. Based on the observation of the graph, many incidents happen on Sunday with 454 cases while wednesday has the smallest cases of fire with 383 cases. This implies that the Bureau of Fire Protection needs to be alert and prepared during the weekends to lessen fire disasters in the province. Figure 5 depicts that the numerous cases of fires are usually happening at the time of 2:00 to 3:00 in the afternoon while their small cases of fire occur in the time of 4:00 in the morning.

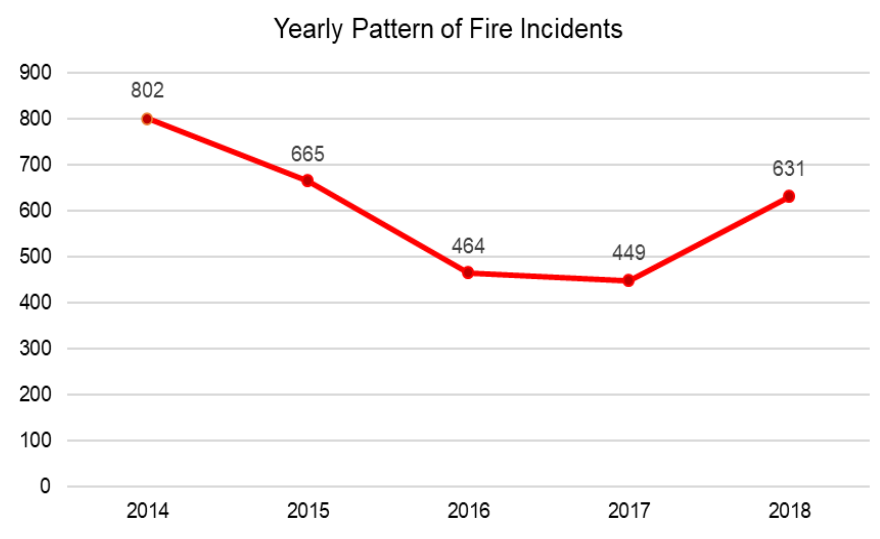

Figure 2. Fire incidents in Laguna per Year since 2014 up-to 2018 


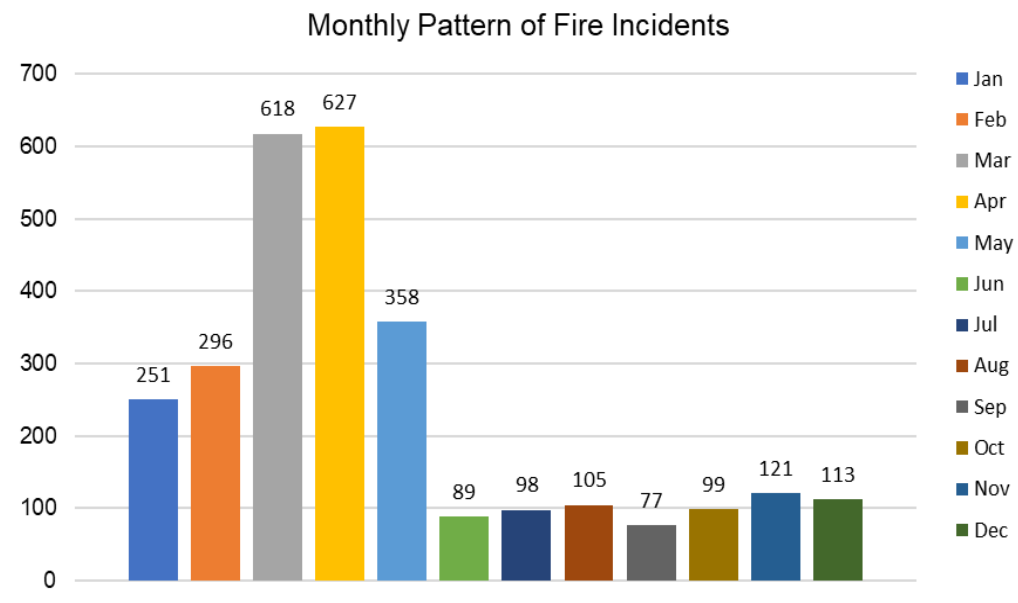

Figure 3. Monthly pattern of fire incidents in Laguna

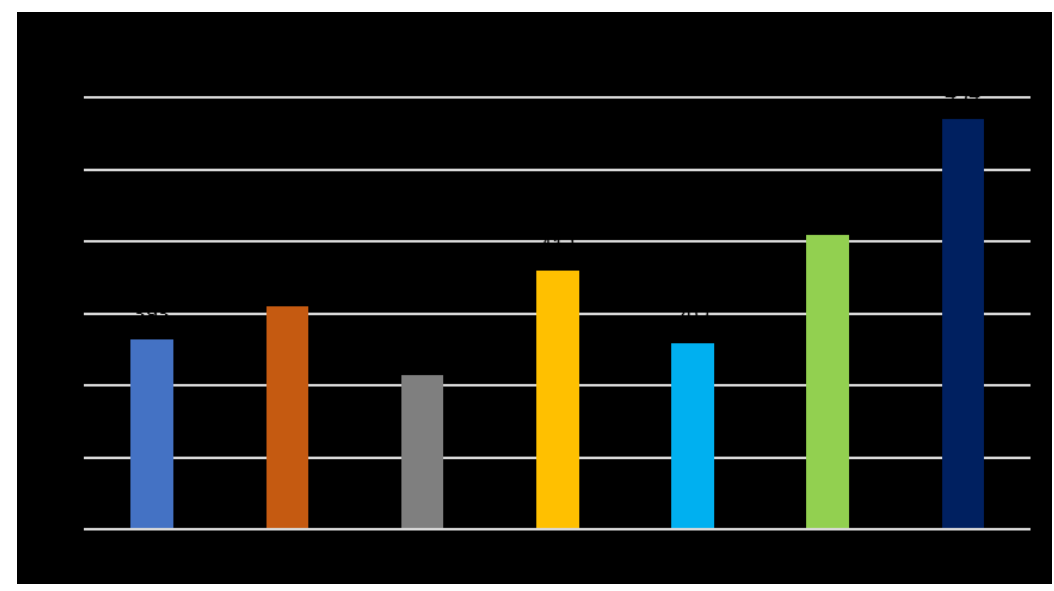

Figure 4. Daily pattern of fire incidents in Laguna

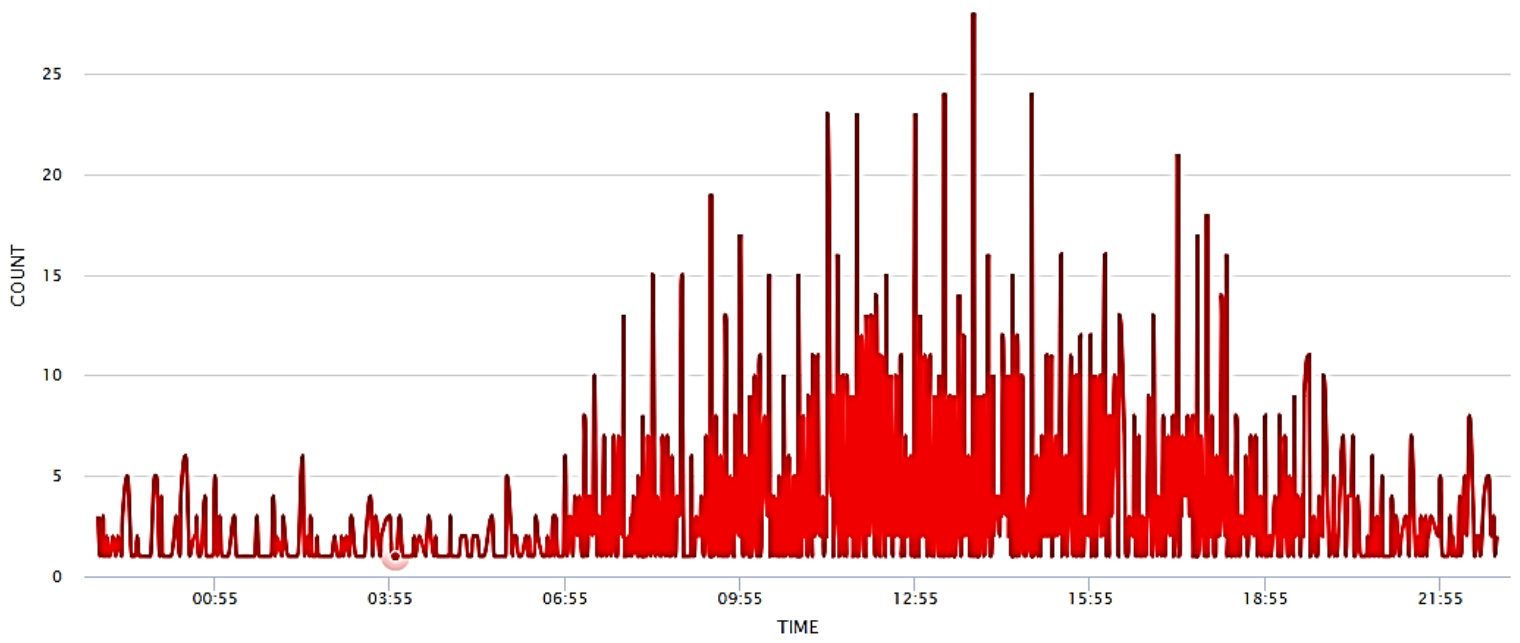

Figure 5. Time pattern of fire incidents in Laguna 
As Figure 6 shows, it is stated that the six cities in Laguna which are Biñan, Cabuyao, Calamba, San Pablo, San Pedro and Sta. Rosa are more likely to have cases of fire incidents because of severe populations in these cities. Among all of the six cities, Calamba City has recorded several incidents in 2014 to 2018 . However, there are only minor cases in the twenty-four remaining municipalities in the province of Laguna.

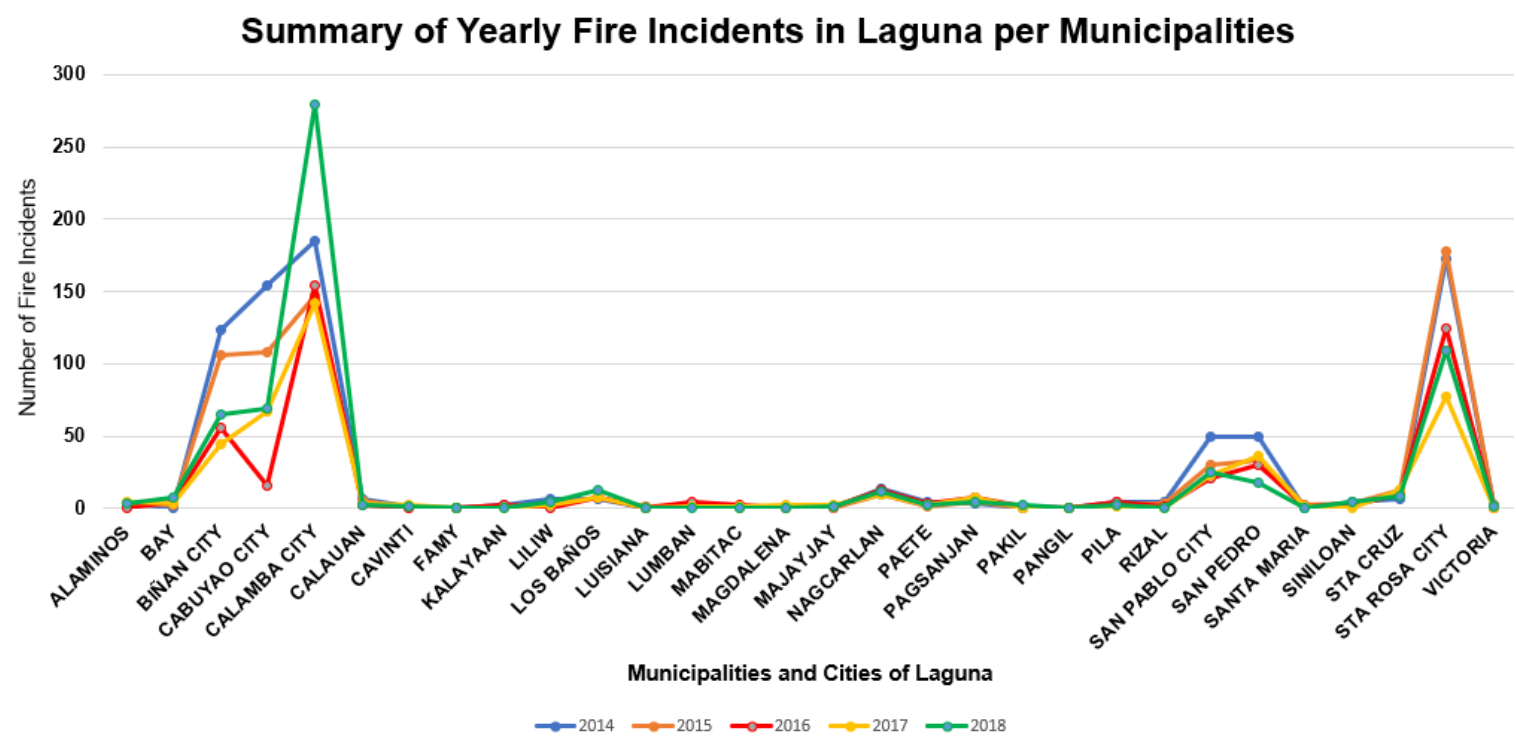

Figure 6. Summary of fire incidents in Laguna per municipality

\section{CONCLUSION}

Among all the machine learning algorithms used in this study, the RNN is noticed to possess a better and precise model. The evaluation of the RNN implies in different parameters and displays an auspicious result choosing the label of alarm levels. It is clear that the use of the algorithm provides a quality pattern in predicting fire incidents in the Province of Laguna. Therefore, recurrent neural network can be used in developing an artificial intelligence model that is capable of learning patterns on how fire occurred in the province of Laguna. Based on the data gathered from the BFP Regional Office Region IV-A, 2014 is listed as the most tragic year in terms of fire occurrences. Also, it is noticed that the month of March and April are the two vulnerable months in fire incidents. Likewise, Sundays, of all days has recorded the greatest number of fires in the province of Laguna, mostly occurring between 2:00 and 3:00 in the afternoon. Moreover, the cities of Laguna are found to have numerous counts of fire incidents, in particular, among these cities, Calamba City has the highest fire incidents recorded. Thus, most of the fire incidents involved is grassfire followed by residential areas.

\section{REFERENCES}

[1] S. Lee, C. Wu, and K. Hung, "A study on fire risks to firefighters in the building with photovoltaic system," 2017 International Conference on Applied System Innovation (ICASI), 2017, pp. 1174-1177, doi: 10.1109/ICASI.2017.7988500.

[2] F. Johnston, et al, "Estimated global mortality attributable to smoke from landscape fires," Environmental Health Perspective, vol. 120, no. 5, pp. 695-701, May 2012, doi: 10.1289/ehp.1104422.

[3] Holborn, Nolan, and Golt, "An analysis of fatal unintentional dwelling fires investigated by London Fire Brigade", Fire Safety Journal, vol. 38, pp. 1-42, Feb. Feb. 2015, doi: 10.1016/S0379-7112(02)00049-8.

[4] Brushlinky, Ahrens, Sokolov, and Wagner, "World Fire Statistics 2015," International Association of Fire and Rescue Services, 2015.

[5] A. Houser, B. Jackson, J. Bartis, and D. Peterson, "Emergency Responder Injuries and Fatalities, in National Personal Protective Technology Laboratory," Santa Monica, CA: RAND Corporation, 2004. [Online]. Available: https://www.rand.org/pubs/technical_reports/TR100.html. Also available in print form.

[6] A. Ramos, "Rural and In-between", Jan. 2015. [Online]. Available: https://www.firefighternation.com

[7] M. Y. Ingal, R. L. T. Tolentino, M. J. Valencia, F. F. Balahadia, and A. R. Caballero, "Fire incidents management system in the city of Manila through geo-mapping," 2016 IEEE Region 10 Symposium (TENSYMP), 2016, pp. 399403, doi: 10.1109/TENCONSpring.2016.7519440. 
[8] C. Siva, "The Difference of Machine Learning and Pattern Recognition," Nov. 2018. [Online]. Available: https://dzone.com/articles/machine-learning-and-pattern-recognition

[9] S. Theodoridis and K. Koutroumbas, "Pattern Recognition 2nd Edition," Greece: Academic Press; An Imprint of Elsevier, 2014

[10] T. M. Cover, "Geometrical and Statistical Properties of Systems of Linear Inequalities with Applications in Pattern Recognition," IEEE Transactions on Electronic Computers, vol. EC-14, no. 3, pp. 326-334, June 1965, doi: 10.1109/PGEC.1965.264137.

[11] M. Y. Orong, A. M. Sison, and A. A. Hernandez, "Mitigating vulnerabilities through forecasting and crime trend analysis," 2018 5th International Conference on Business and Industrial Research (ICBIR), May 2018, pp. 57-62, doi: 10.1109/ICBIR.2018.8391166.

[12] X. Lagorce, G. Orchard, F. Galluppi, B. E. Shi, and R. B. Benosman, "HOTS: A hierarchy of event-based timesurfaces for pattern recognition," IEEE Transactions on Pattern Analysis and Machine Intelligence, vol. 39, no. 7, pp. 1346-1359, Jul. 2017, doi: 10.1109/TPAMI.2016.2574707.

[13] J. B. Lani, "What is Logistic Regression,” Jun. 2015. [Online]. Available: https://www.statisticssolutions.com/whatis-logistic-regression/

[14] G. Amatulli, M. J. Rodrigues, M. Trombetti, and R. Lovreglio, "Assessing Long-term Fire Risk at Local Scale by Means of Decision Tree Technique," Journal of Geophysical Research Atmospheres, pp. 65-67, Dec. 2016, doi: 10.1029/2005JG000133.

[15] J. M. Saveland and L. F. Neuenschwander, "A Signal Detection Framework to Evaluate Models of Tree Mortality Following Fire Damage," International Journal of Forest Science, pp. 98-100, 2017, doi: 10.1093/forestscience/36.1.66.

[16] J. R. Asor and M. A. T. Subion, "RESEARCH++: An academic social networking research community portal for profiling and expertise classification," 2018 International Seminar on Research of Information Technology and Intelligent Systems (ISRITI), 2018, pp. 470-475, doi: 10.1109/ISRITI.2018.8864483.

[17] G. M. B. Catedrilla and J. R. Asor, "Pattern Recognition from Radiology Reports towards Predictive Lung Disease Manifestation in Municipal Settings," 2018 International Seminar on Research of Information Technology and Intelligent Systems (ISRITI), 2018, pp. 476-480, doi: 10.1109/ISRITI.2018.8864241.

[18] J. R. Asor, G. M. B. Catedrilla, and Jefferson L. Lerios, "Usage of classification algorithm for extracting knowledge in cholesterol report towards non-communicable disease analysis," Journal of Advances in Information Technology, vol. 11, no. 4, pp. 265-270, Nov. 2020, doi: 10.12720/jait.11.4.265-270.

[19] J. R. Asor, G. M. B. Catedrilla, and J. E. Estrada, "A study on the road accidents using data investigation and visualization in Los Baños, Laguna, Philippines," 2018 International Conference on Information and Communications Technology (ICOIACT), 2018, pp. 96-101, doi: 10.12720/jait.11.4.265-270.

[20] J. R. Asor and S. B. Sapin, "Implementation of Predictive Crime Analytics in Municipal Crime Management System in Calauan, Laguna, Philippines," International Journal of Advanced Trends in Computer Science and Engineering, vol. 9, no. 1.3, pp. 150-157, Jun. 2020, doi: 10.30534/ijatcse/2020/2291.32020.

[21] J. Lerios and M. Villarica, "Pattern Extraction of Water Quality Prediction Using Machine Learning Algorithms of Water Reservoir," International Journal of Mechanical Engineering and Robotics Research, vol. 8, no. 6, pp. $992-$ 997, 2019, doi: 10.18178/ijmerr.

[22] N. Chawla, K. Bowyer, L. Hall, and P. Kegelmeyer, "SMOTE: Synthetic minority over-sampling technique", Journal of Artificial Intelligence Research, vol. 16, no. 1, pp. 321-324, Jun. 2002, doi: 10.1613/jair.953.

[23] A. Mishra, "Metrics to evaluate your machine learning algorithm," Feb. 2018. [Online]. Available: https://towardsdatascience.com/metrics-to-evaluate-your-machine-learning-algorithm-f10ba6e 38234

[24] G. I. Webb, "Algorithm evaluation, in encyclopedia of machine learning," Boston, Springer, 2015, pp. 45.

[25] F. F. Balahadia, J. R. Asor, G. M. B. Catedrilla, M. Villarica, and J. M. Cabiente, "Intelligent Investigation on Crime Incident Reports in the Province of Laguna through Predictive Model Development," International Journal of Advanced Trends in Computer Science and Engineering, vol. 9, no. 1.3, pp. 139-144, Jun. 2020, doi: 10.30534/ijatcse/2020/2091.32020.

[26] M. Niwariya, A. Rajput, and S. Jaloree, "Data Mining Approach for Diabetes Prediction using BPSO, SVM, KNN and Naïve Bayes Classifiers," International Journal of Advanced Trends in Computer Science and Engineering, vol. 9, no. 1.5, pp. 286-293, Sept. 2020, doi: 10.30534/ijatcse/2020/4191.52020.

[27] E. R. Bondoc, et al., "An intelligent road traffic information system using text analysis in the most congested roads in Metro Manila," 2018 IEEE 10th International Conference on Humanoid, Nanotechnology, Information Technology, Communication and Control, Environment and Management (HNICEM), 2018, pp. 1-6, doi: 10.1109/HNICEM.2018.8666416. 


\section{BIOGRAPHIES OF AUTHORS}
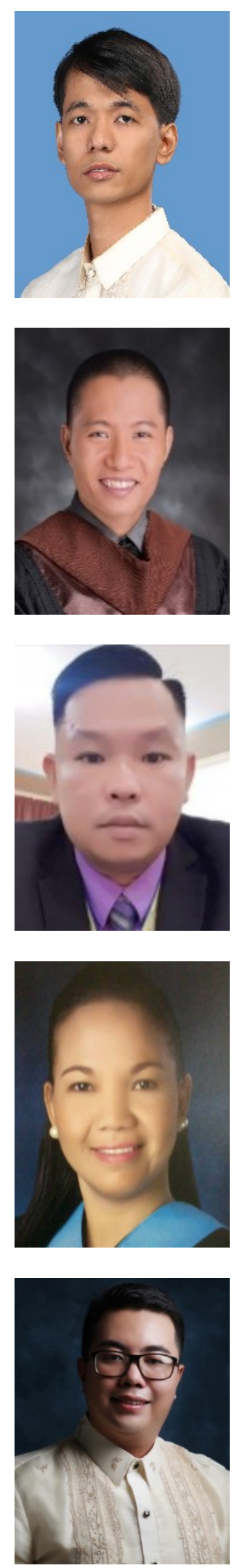

Jonardo R. Asor was born in Los Baños, Laguna, Philippines on June 12, 1990. He graduated at the Laguna State Polytechnic University, Los Baños campus with the degree bachelor of science in information technology on March 2016. Mr. Asor finished his master in information technology at the Technological Institute of the Philippines, Manila, on October 2018. He is currently working as College Instructor at the Laguna State Polytechnic University, Los Baños Campus, Malinta, Los Baños, Laguna, Philippines. His research study focuses on the optimization and usage of machine learning algorithm and knowledge discovery in databases.

Jefferson L. Lerios is a graduate of Doctor of Information Technology at the Technological Institute of the Philippines Manila. Currently, he is an Assistant Professor II at the College of Computer Studies, Laguna State Polytechnic University, Los Baños Campus. His research interest is in the field of environmental modeling, intelligent systems, information technology, data mining, and data analytics.

Dr. Sherwin B. Sapin is currently the Chairperson of the Curriculum and Instruction Development and the Coordinator for Local Government Unit of Laguna State Polytechnic University-Los Baños Campus. His research interests are in Information Technology Education, Mathematics Education, and Educational Management. He authored various research studies published in different international journals.

Jocelyn O. Padallan is a graduate of Master of Arts in Education major in Educational Management at the Laguna State Polytechnic University, Los Baños. Currently, she is an Assistant Professor II at the College of Computer Studies, Laguna State Polytechnic University, Los Baños Campus and affiliated as adviser of CCS-Student council. H research interest is in the field of information technology, software development and data analytics.

Dr. Chester Alexis C. Buama is a College Professor at Laguna State Polytechnic University under the Faculty of College of Arts and Sciences. A Consultant of the Department of Education Central Office for Learning Assessment and National Trainer of the Commission on Higher Education for General Education Courses. He is also a member of local and international organizations: he is a Fellow and Senior Member of the Royal Institute of Management and the Royal Institute of Educators, Singapore, Reading Association of the Philippines, and International English Learners Training Institute. He has a Master's degree in Management major in Public Administration and a Bachelor's degree in Journalism. He was conferred a Doctorate Degree in Philosophy major in Public Administration in 2016 and graduated with a Doctorate Degree in Philosophy major in Business Management in 2018. His research interest is in Social Science, and Business Management. 